\title{
The Design and Operation of Infrasonic Microphones
}

\author{
J. Paul Mutschlecner \\ Rodney W. Whitaker
}

\section{DISCLAIMER}

This report was prepared as an account of work sponsored by an agency of the United States Government. Neither the United States Government nor any agency thereof, nor any of their employees, makes any warranty, express or implied, or assumes any legal liability or responsibility for the accuracy, completeness, or usefulness of any information, apparatus, product, or process disclosed, or represents that its use would not infringe privately owned rights. Reference herein to any specific commercial product, process, or service by trade name, trademark. manufacturer, or otherwise does not necessarily constitute or imply its endorsement, recommendation, or favoring by the United States Government or any agency thereof. The views and opinions of authors expressed herein do not necessarily state or reflect those of the United States Government or any agency thereof. 


\title{
The Design and Operation of Infrasonic Microphones
}

by

\author{
J. Paul Mutschlecner and Rodney W. Whitaker
}

\begin{abstract}
This report is intended as a guide to the design and operation of infrasonic microphones. It will emphasize general principles and the effects of parameter choices upon performance but will not provide details of design for specific microphones. The report will consider primarily the mechanical aspects that control the acoustic properties; it will not discuss the features of electronic design, which vary greatly among microphones. Here we define infrasonic microphones as sensors capable of detecting pressure variations in the period range of about $0.1 \mathrm{~s}$ to $1000 \mathrm{~s}$ with changes from about $0.1 \mu$ bar (microbar $=1$ dyne $\mathrm{cm}^{-2}$ ) to about 1 mbar.
\end{abstract}

\section{GENERAL DESIGN}

Figure 1 presents a schematic of the design of a typical infrasonic microphone. A fore volume $(V)$ receives a pressure variation from the outside through a connection $(R)$. The pressure variation is communicated to a backing volume $(v)$ through a combination of the leak $(r)$ and the flexible diaphragm $(b)$. The ambient pressure is designated by $P_{a}$ and the signal incremental pressure by $P_{e}$. The incremental pressure inside the fore volume is $P$, and the incremental pressure in the backing volume is $p . P$ and $p$ are not, in general, equal to $P_{e}$ due to the effects of the elements $R, r$, and $b$. We are interested in the differential pressure across the diaphragm, $P-p$, since this is what is sensed by the microphone. Infrasonic microphones may take other forms. For example, Jones and Forbes (1962) discuss a double bellows design, and Haak and de Wilde (1996) discuss an absolute pressure bellows design. 


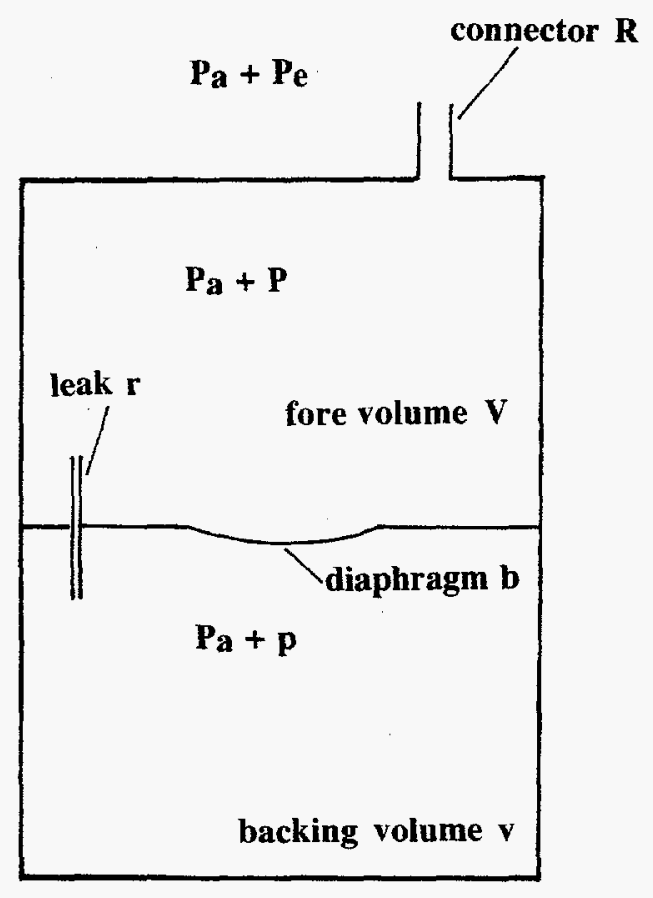

Figure 1. Schematic design of an infrasonic microphone. The individual components are described in the text.

\section{ACOUSTIC RESISTANCE}

Both the connection to the outside and the leak between the two volumes can be characterized by their acoustic resistance, which determines the rate at which volume flow can occur under a given pressure differential. Thus we have

$$
R_{a}=\Delta P /(d V / d t)
$$

where $R_{a}$ represents acoustic resistance, $d V / d t$ a rate of volume transfer through a channel, and $\Delta P$ the pressure differential between the ends of the channel. For a cylindrical channel, the acoustic resistance can be estimated by the Hagen-Poiseuille relation:

$$
R_{a}=8 L \eta / \pi a^{4}
$$

where $L$ is the length of the channel, $\eta$ the molecular shear viscosity of air, and $a$ the radius of the channel. The value $\eta$ is a function of air temperature, but a reasonable average value can be taken as $1.8 \times 10^{-4}$ poise (poise $=\mathrm{g} \mathrm{cm}^{-1} \mathrm{~s}^{-1}$ ). The acoustic resistance is often stated in ohms $(\mathrm{ohm}=\mathrm{g}$ $\mathrm{cm}^{-4} \mathrm{~s}^{-1}$ ). The applicability of the formula (2) is dependent upon several conditions including 


\section{DISCLAIMIER}

Portions of this document may be illegible in electronic image products. Images are produced from the best available original document. 
laminar flow within the channel and the absence of significant end effects on the channel; these conditions should be satisfied here.

The connection of the fore volume to the outside has an acoustic resistance $R$. In the case of a "bare" microphone, the connection may be a short pipe or open port, and the value of $R$ will be very small. If the microphone is connected to some type of noise reducer, e.g., a pipe noise reducer of the type described by Daniels (1959) and others, the effective $R$ may be much larger. The value of $R$ will substantially control the high-pass (high-frequency) cutoff of the microphone.

The leak connection between the fore volume and the backing volume has an acoustic resistance $r$. Typically the leak is composed of a length of very small-bore capillary tubing or an adjustable hypodermic needle valve. The value of $r$ is very large compared with $R$ and has primary control over the low-pass cutoff of the microphone. The essential function of this leak is to permit the pressure in the backing volume always to be close to $P_{a}$, the ambient pressure, so that the diaphragm need only be sensitive to microbaric changes and not to the larger millibaric changes, which occur over relatively long periods (e.g., as caused by weather changes).

\section{THE DIAPHRAGM}

The diaphragm, together with its electrical coupling, compose the sensing component of the microphone. The diaphragm is a flexible membrane, or in some designs a bellows, which can move in response to the pressure differential, $P-p$. Typically, the motion of the diaphragm is measured by an electrical coupling, such as a condenser, of which the diaphragm is an element. In turn, the condenser is an electronic circuit component that may, for example, control a modulation on an FM carrier and, thence, a voltage change. In an ideal microphone the voltage change will be linearly proportional to the signal pressure $P_{e}$. Hubbard and Bedard (1969) suggest other desired characteristics of an infrasonic microphone. We characterize the action of the diaphragm (or bellows) by

$$
b=\Delta V / \Delta P
$$

where $b$ is the diaphragm constant, $\Delta P$ the pressure differential across the diaphragm, and $\Delta V$ a volume change produced by the corresponding motion of the diaphragm. A downward expansion of the diaphragm in Figure 1 will result in an increase in $V$ in the fore volume and a decrease in $v$ in the backing volume. Note that corresponding to the very small differential pressures, extremely small motions of the diaphragm will occur (e.g., fractions of a $\mu \mathrm{m})$. It is vital that the container for the backing volume be very rigid so that volume changes are produced only by the action of the diaphragm. 


\section{PRESSURE INCREMENTS}

We now examine the roles of the microphone elements in determining the pressure increments in each of the volumes. The time rate of change of the incremental pressure $P$ in the fore volume is given by

$$
\frac{d P}{d t}=\left(\frac{d P}{d t}\right)_{R}+\left(\frac{d P}{d t}\right)_{r}+\left(\frac{d P}{d t}\right)_{b},
$$

where the subscripts denote the dependencies of each term; the terms represent, respectively, the effects of the inlet resistance, the leak resistance, and the diaphragm. Similarly, the rate of change for the backing volume will be given by

$$
\frac{d p}{d t}=\left(\frac{d p}{d t}\right)_{r}+\left(\frac{d p}{d t}\right)_{b}
$$

where the terms on the right represent in turn the effects of the leak and the diaphragm. The individual terms can now be replaced with the use of (1) and (3). We use an adiabatic approximation to the gas law to replace the volume differentials by pressure differentials. For example,

$$
\Delta P=-\gamma P_{a} \Delta V / V,
$$

where $\gamma$ is the adiabatic gas constant $(\gamma=1.401)$. The adiabatic approximation is made under the assumption that the construction of the microphone is such that all processes are not instantaneously isothermal. Pierce (1981) and others indicate the general applicability of the adiabatic approximation in situations such as these. Obviously for cases where the isothermal approximation may be more appropriate, $\gamma$ is removed from (6).

We can now rewrite (4) and (5) as

$$
\frac{d P}{d t}=\frac{\gamma P_{a}}{V}\left[\frac{P_{e}-P}{R}+\frac{p-P}{r}+b \frac{d(p-P)}{d t}\right]
$$

and

$$
\frac{d p}{d t}=\frac{\gamma P_{a}}{v}\left[\frac{P-p}{r}+b \frac{d(P-p)}{d t}\right] .
$$

These expressions can be used to determine the time dependencies $P(t)$ and $p(t)$, given a time dependence of the pressure at the entrance port $P_{e}(t) . P_{e}(t)$ is simply the local pressure 
perturbation (i. e., signal) for a bare microphone but will result from a complex conditioning of the signal in the case where a noise reducer system is attached at the entrance port.

\section{MICROPHONE RESPONSE}

We now define the response function, $Q$, of the microphone by

$$
Q=\langle P-p\rangle /\left\langle P_{e}\right\rangle
$$

where the brackets represent root-mean-square values. This is a statement that the microphone can only respond to the differential P-p and thus only to some fraction, $Q$, of the signal $P_{e}$. This response is frequency dependent. Note that published response functions are often normalized such that the maximum is set to unity. In order to examine the response function for $P_{e}(t)$ as a continuous sine wave, we write

$$
\begin{aligned}
& P_{e}=e^{i \omega t}, \\
& P=m e^{i \omega t},
\end{aligned}
$$

and

$$
p=n e^{i \omega t}
$$

where the amplitude for $P_{e}$ is taken as unity, and we indicate that $P(t)$ and $p(t)$ will be forced as sine wave forms but with amplitudes reduced by the factors $m$ and $n$, respectively. In this substitution we have converted the pressures to complex quantities; $m$ and $n$ are also complex. This follows a conventional procedure for the analysis of oscillatory behavior. Here $\omega$ is the angular frequency given by

$$
\omega=2 \pi / T,
$$

where $T$ is the period. Substituting (10) through (12) into (9) yields the simple expression

$$
Q=m-n
$$

A solution for $m-n$ thus leads to a determination of the complex response function. This derivation is carried out in Appendix A. Because the expression for $Q$ is a complex number, we find the amplitude of $Q, \Re$, as the final expression of microphone response, and we henceforth refer to $\mathfrak{R}$ as the response. The result found in Appendix $\mathrm{A}$ is 


$$
\Re=\sqrt{Q Q^{*}}=\frac{\omega a v}{\sqrt{C^{2}+D^{2}}}
$$

where

$$
C=\frac{a^{2}}{r}-\omega^{2} R[V v+a b(V+v)]
$$

and

$$
D=\omega a\left[a b+v+\frac{R}{r}(V+v)\right]
$$

where $\mathrm{a}$ is $\gamma P_{a}$. In addition the solution (see Appendix A) provides a determination of the phase, $\phi$, of $P$ - $p$ with respect to the signal wave $P_{e}$ :

$$
\tan \phi=\frac{a-\omega^{2} r R[b(V+v)+V v / a]}{\omega[r(v+a b)+R(V+v)]}
$$

A useful approximation to (18) derived in Appendix A is

$$
\tan \phi \approx \frac{a}{\omega g}-\frac{\omega R V}{a}
$$

where

$$
g=r(a b+v)
$$

From (19) the frequency, $\omega_{c}$, for null phase lag is

$$
\omega_{c}=a / \sqrt{R V g}
$$

\section{NUMERICAL INTEGRATION}

Another procedure for determination of the response is the numerical integration of expressions (7) and (8) to obtain $P(t)$ and $p(t)$. In this procedure the signal function $P_{e}(t)$ can be expressed as a sine wave or as some other time-dependent function. Unlike the analytic procedure, the numerical integration in small time steps simulates a microphone's physics and is carried out in real numbers. We performed numerical integrations for several simulations, which give further insight on microphone physics and, with the analytic method, provide mutual confirmation of the results. Figure 2 illustrates the functions $P(t)$ and $p(t)$ that result from a numerical integration for a standard example, described below, with a sine wave input signal having a frequency 0.628 rad s ${ }^{-1}$. The amplitudes $M$ and $N$ of $P$ and $p$, respectively, are given by the simulations and are the real-number amplitudes corresponding to the complex quantities $m$ and $n$. In addition, the phase, 
$\chi$, between $P$ and $p$ is determined from the simulation; in Figure 2 it is seen that $p$ is advanced in phase with respect to $P$.

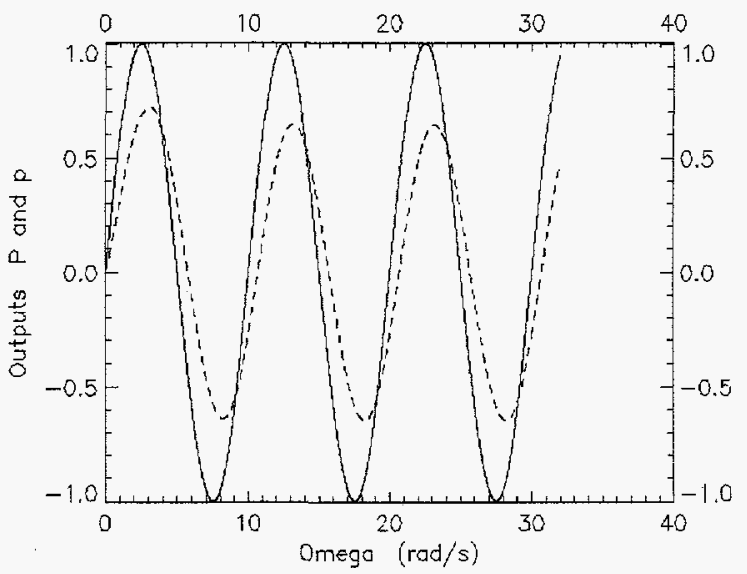

Figure 2. Illustration of the results of a numerical integration for a microphone's response. The curves show the pressure $P$ (solid line) in the fore volume and $p$ (dashed line) in the backing volume for a driving pressure with a period of 10 seconds. It can be seen that there is a phase lag of $p$ over $P$.

The quantities obtained from a numerical simulation can be connected to the analytic results described earlier. Thus we can write for the response

$$
\Re=\left(M^{2}-2 M N \cos \chi+N^{2}\right)^{1 / 2}
$$

The phase, $\phi$, as defined by (18) is related to the numerical integration phase, $\chi$, by

$$
\tan \phi=\frac{N \sin \chi}{M-N \cos \chi}
$$

The results from (22) and (23) give excellent agreement with the analytic results for the simulations that we performed.

\section{STANDARD CASE-AN EXAMPLE}

As an illustration we predict the response for a microphone with an assumed set of parameters: 


$$
\begin{aligned}
R & =0.4 \mathrm{ohms} \\
r & =10^{4} \mathrm{ohms} \\
v & =200 \mathrm{~cm}^{3} \\
V & =4 \times 10^{4} \mathrm{~cm}^{3} \\
b & =10^{-4} \mathrm{~cm}^{5} \text { dyne }^{-1}
\end{aligned}
$$

These values are representative of a typical, moderate-sized field microphone that is attached to a noise reducer. We assume a noise reducer consisting of eight pipes of 20-meter length with inlet ports spaced at 0.3 meter. The microphone leak is assumed to be a long capillary tube (length $\mathbf{5 0}$ $\mathrm{cm}$ and diameter $0.04 \mathrm{~cm}$ ), which results in the high resistance $r$. The backing volume is a small cylinder. The diaphragm parameter, $b$, can be no larger than that which will produce equilibrium following an incremental increase in the value of $P$, assuming that the diaphragm is passive and has no mechanical advantage. Using (3) and (6) we find that this requires

$$
b \leq v / \gamma P_{a} .
$$

For the standard case this gives $b<1.43 \times 10^{-4} \mathrm{cgs}$ and we adopt $10^{-4} \mathrm{cgs}$. Figure 3 shows the response curve for this standard case. The maximum response is about 0.59 , and the microphone has low- and high-pass power 3-dB points of about $0.41 \mathrm{rad} \mathrm{s}^{-1}$ and $88 \mathrm{rad} \mathrm{s}^{-1}$, respectively. In general, the high-frequency response may be reduced by a high-pass electronic filter. An expression for the frequency, $\omega_{\mathrm{m}}$, in which the response is maximum, is obtained through the operation

$$
\frac{\partial \mathfrak{R}}{\partial \omega}=0
$$

Using (15) through (17) the result is

$$
\omega_{m}=\frac{a}{\sqrt{\operatorname{RrV(v+ab)}}}
$$

The frequency for maximum response in the standard example is $6.01 \mathrm{rad} \mathrm{s}^{-1}$. Note that the expression for $\omega_{\mathrm{m}}$ is identical to that for null phase (21). Now $\mathfrak{K}_{m}$, the maximum response, can be found by using $\omega_{\mathrm{m}}$ in (15) through (17). For the standard case the result is 0.588 . In Figure 4 the relation between the phase lag and frequency is shown for the standard case. It is seen that $\phi$ can be positive or negative and is asymptotic to $90^{\circ}$ or $-90^{\circ}$ at very low or high frequencies. 


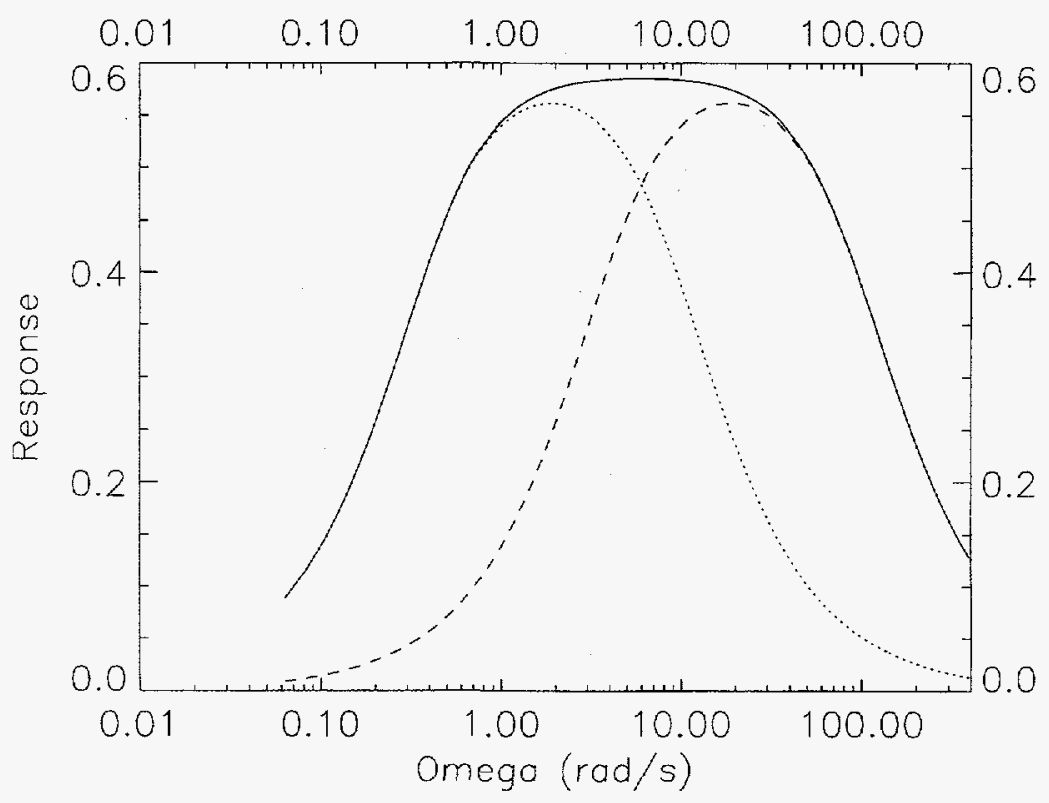

Figure 3. Response of a microphone versus frequency for the standard case described in the text (solid line). The figure also illustrates the effect of an increase in the leak resistance, $r$, by a factor of ten (dashed line) and an increase in $R V$ by a factor of ten (dotted line).

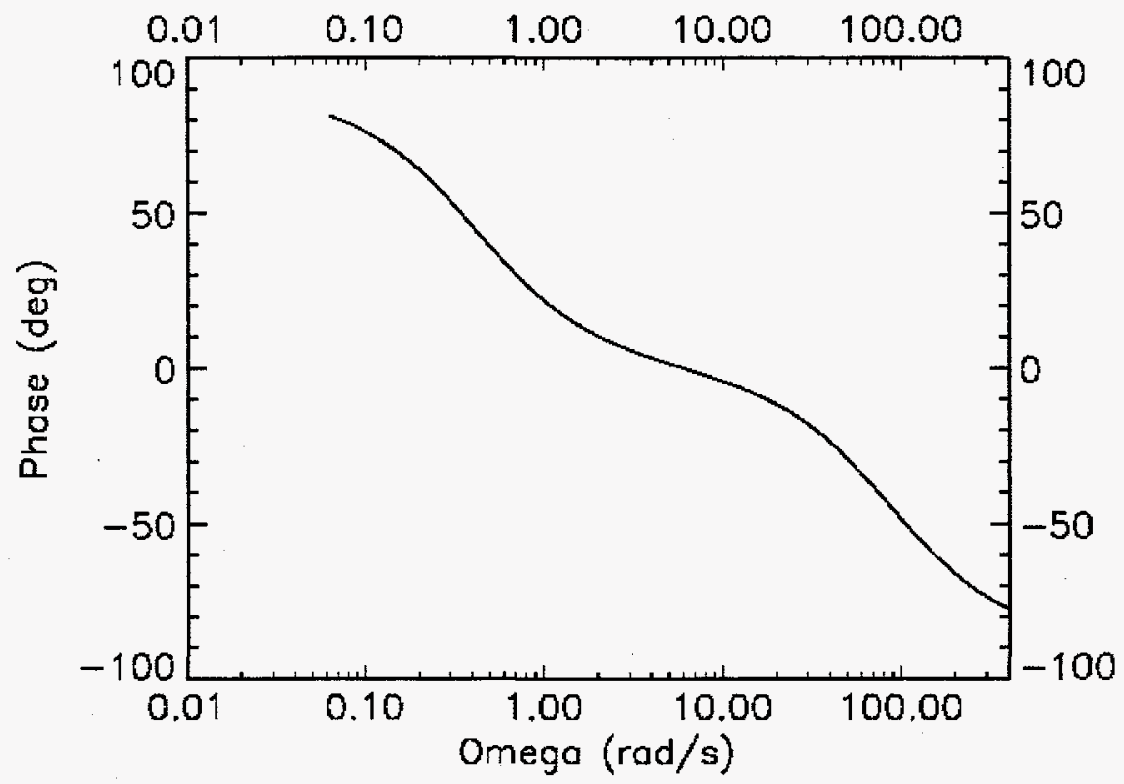

Figure 4. The phase lag $\phi$ of the pressure $P-p$ with respect to $P_{e}$ versus frequency for the standard case given in the text. 
Note that in this example we have made only a rough estimate of the effect of the noise reducer through the parameters $R$ and $V$. In fact, the effects of a noise reducer must be considered separately since it independently conditions the signal. In treating an entire system, the quantity $P_{e}$ at the microphone is the signal as modulated by the noise reducer.

\section{PARAMETER EFFECTS}

\section{A. The Influence of $r$}

The parameter $r$ has primary control of the low-pass (low-frequency) part of the response. We can approximate this portion of the response by examining the relative importance of terms in (16) and (17) and dropping those that are minor for the frequency domain $\omega<1$. The same result is obtained by setting $R=0$. This has the effect of opening the fore volume directly to the signal $P_{e}$; only the leak, $r$, then has control of the frequency response and, because of its very high value, it controls long-period response. The resulting approximation for the low-pass response is

$$
\mathfrak{R}_{l} \approx \frac{v}{\sqrt{(a / \omega r)^{2}+(a b+v)^{2}}}
$$

We can determine the low-pass power $3-\mathrm{dB}$ point frequency, $\omega_{l}$, by solving

$$
\mathfrak{R}_{l}\left(\omega_{i}\right)=\mathfrak{K}_{m} / \sqrt{2},
$$

and this yields

$$
\omega_{l}=\frac{a}{r}\left[\left(\frac{\sqrt{2} v}{\mathfrak{R}_{m}}\right)^{2}-(a b+v)^{2}\right]^{1 / 2} .
$$

In the standard case the result is $0.413 \mathrm{rad} \mathrm{s}^{-1}$. In Figure 3 we illustrate the effect of $r$ on the response curve with a change in value to $10^{3} \mathrm{ohms}$. The response to lower frequencies is decreased as suggested by (29).

The action of a leak and its associated volume is sometimes expressed in terms of a time constant. If we integrate (8) with no diaphragm influence, we can write

$$
p=p_{o} \exp (-t / K)
$$

where $p_{o}$ is an initial value of $p$, and $K$ is given by 


$$
K=r v / \gamma P_{a} .
$$

$K$ is referred to as the time constant of the $r-v$ system. An electrical analog can be given as

$$
K=r c
$$

where $r$ is the resistance of the leak, and $c$ is a "capacitance" defined by

$$
c=v / \gamma P_{a} .
$$

For the standard case $K=5.71 \mathrm{~s}$.

\section{B. The Influence of $R V$}

Notice in (16) and (17) that the parameters $R$ and $V$ always occur as a pair. $R V$ has the primary control over the high-pass part of the response. We can approximate the high-pass response, $\mathfrak{h}_{h}$, by eliminating terms which become unimportant for $\omega>100 \mathrm{rad} \mathrm{s}^{-1}$ or equivalently setting $r=\infty$. The later condition has the effect of closing the backing volume except for the influence of the diaphragm so that the very short-period influence of $R V$ is emphasized. The result is

$$
\mathfrak{K}_{h} \approx \frac{v}{(a b+v) \sqrt{\frac{\omega R V}{a}+1}} .
$$

Using the equivalent of (28) for high-pass, we can find the 3-dB point frequency for high-pass, $\omega_{h}$, as

$$
\omega_{h}=\frac{a}{R V(v+a b)} \sqrt{\left(\frac{\sqrt{2} v}{\Re_{m}}\right)^{2}-(a b+v)^{2}} .
$$

For the standard case this gives $87.3 \mathrm{rad} \mathrm{s}^{-1}$. Figure 3 shows the effect of an increase of $R V$ from the standard case value of $1.6 \times 10^{4}$ to $1.6 \times 10^{5} \mathrm{cgs}$. We see the reduction of high-frequency response. For the $R-V$ system in isolation we have $K=875 \mathrm{~s}$.

\section{The Influence of $\boldsymbol{b}$}

The diaphragm constant, $b$, primarily provides control over the absolute sensitivity of the microphone. As $b$ decreases, the response increases. For $\mathrm{b}=0$, the response is unity, but, of course, there is no microphone signal because the diaphragm does not move. In Figure 5 we show 
the effect of $b$ by comparison of the response for the standard case $\left(b=10^{-4} \mathrm{cgs}\right)$ with that for $b=$ $5 \times 10^{-5} \mathrm{cgs}$. The figure shows the primary influence of $b$ on the peak region of the response; however, $b$ also influences the high-frequency region.

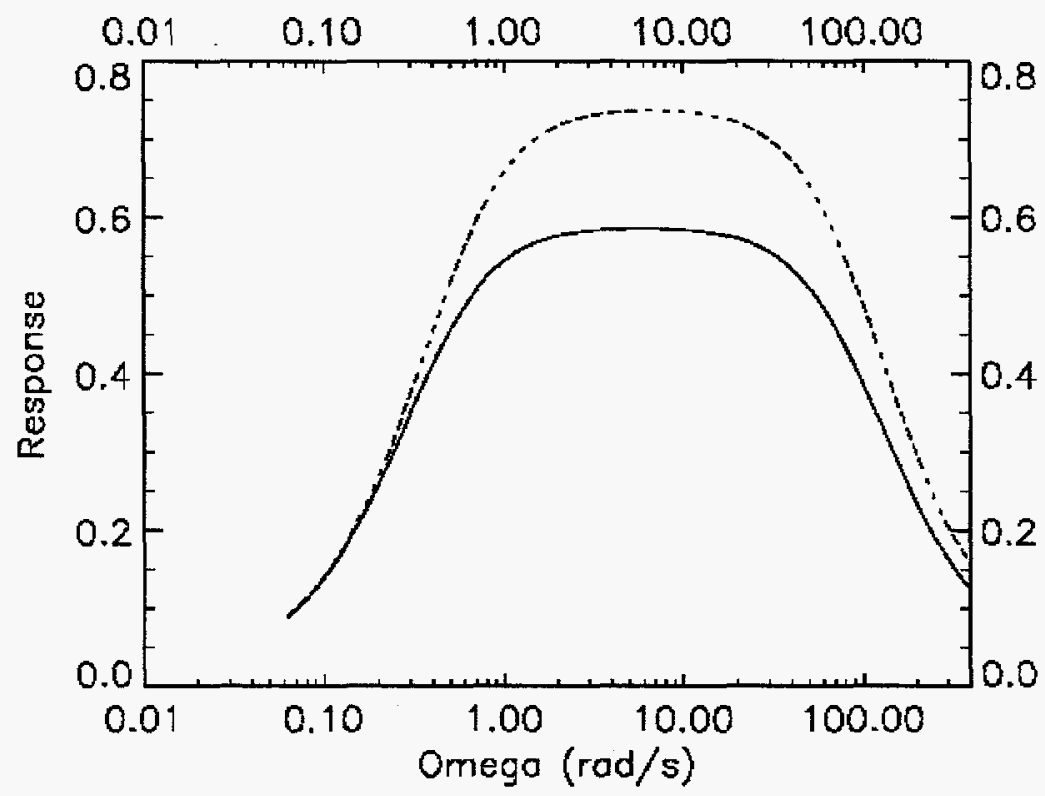

Figure 5. The response of a microphone for the standard case (solid line) compared with the response for a decrease in the diaphragm parameter, $b$, by a factor of two (dashed line).

\section{PHASE EFFECTS}

The phase, $\phi$, given by (18) or (19) is normally not important or noticeable for single microphone operation. However, when microphones are operated in an array, the effects of the phase lag must be considered. The relative arrival times of a signal across an array provide a determination of the trace velocity, $V_{t}$, which is the horizontal component of the signal speed across the array, and of the azimuth of the signal, $\Theta$. If the microphones do not have a common value of $\phi$, there will be errors in the determination of $\Theta$ and $V_{t}$. From (19), by partial differentiation, we can determine the influence of errors in the microphone parameters upon $\phi$. We consider the effects of errors $\delta r, \delta R V$, and $\delta b$, in $r, R V$, and $b$, respectively, in producing an error $\delta \phi:$

$$
\delta \phi=-\frac{a}{\omega r g\left(I+\tan ^{2} \phi\right)} \delta r,
$$




$$
\delta \phi=-\frac{\omega}{a\left(1+\tan ^{2} \phi\right)} \delta R V
$$

and

$$
\delta \phi=-\frac{a^{2} r}{\omega^{2}\left(1+\tan ^{2} \phi\right)} \delta b
$$

We evaluate these expressions for the standard case with $\phi=0$ (this gives the maximum value of the effect) with $10 \%$ errors in the parameters. The result is that $|\delta \phi|$ is less than about $0.3^{\circ}$ for all parameters. Thus substantial errors must be made in the parameters to produce an appreciable error in $\phi$. We can estimate the maximum effects upon $V_{t}$ and $\Theta$ by considering a pair of microphones on a line radial to the signal direction and tangential to the signal direction, respectively. Figure 6 illustrates the geometry. For an accurate evaluation, the details of a specific array design must be considered. We find for the example of the two microphones shown in Fig. 6 the errors in $V_{t}$ and $\Theta$ :

$$
\delta V_{t}=\frac{V_{t} \Delta \phi}{180}
$$

and

$$
\delta \theta=-\frac{\Delta \phi}{\pi}
$$

where $\Delta \phi$ is the phase of sensor A with respect to sensor B expressed in degrees, $\delta \theta$ is in degrees, and we assume that the spacing of the microphones is an optimum one-half wavelength. The results from (39) and (40) for the standard case are:

$$
\delta V_{t} \approx 0.75 \mathrm{~ms}^{-1}
$$

and

$$
\delta \theta \approx 0.1^{\circ}
$$

Fortunately, in general, large errors in phase would be needed to produce significant errors in the trace velocity or azimuth. 
(a) Phase Effects on $V$,

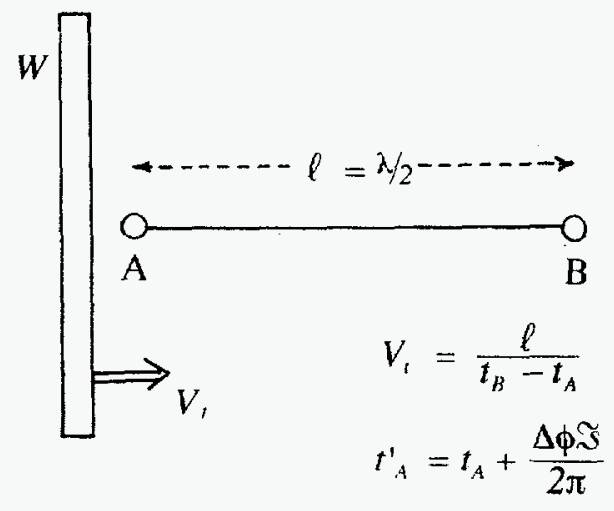

(b) Phase Effects on $\theta$

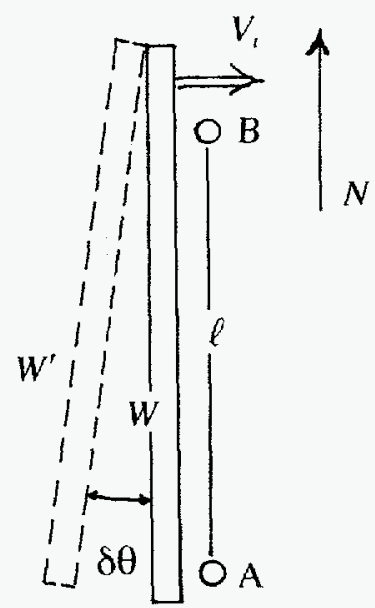

$$
\theta \approx \frac{3 \pi}{2}+\frac{\left(t_{A}-t_{B}\right) V_{t}}{\ell} \quad(\mathrm{rad})
$$

Figure 6. This illustrates the geometry for an estimation of the effects of a phase lag on the determination of (a) trace velocity, $V_{t}$, and (b) azimuth, $\Theta$. The figures show plan views of a pair of microphones $\mathrm{A}$ and $\mathrm{B}$ separated by distance $\ell$ with an approaching plane wave front $\mathrm{W}$ traveling at speed $V_{t .}$. We assume that microphone $\mathrm{A}$ has a phase error, $\Delta \phi$, which changes the true arrival time $t_{A}$ to $t_{A}^{\prime}$. The symbol $\Im$ represents the period. In $\mathrm{b}$ the fictitious wave front $\mathrm{W}$ ' results from the phase error and produces a increase of azimuth by $\delta \theta$.

\section{SEISMIC SENSITIVITY}

Infrasonic microphones are designed for detection of pressure perturbations transmitted directly through the atmosphere to the microphone. However, microphones may also be sensitive to distant disturbances transmitted through seismic motion of the earth. This may happen by three 
means: (1) Traveling seismic waves may produce a coupled surface acoustic wave which is detected by the microphone. (2) The seismic motion of the earth is communicated to the microphone as a vertical oscillation, which is then detected as we describe later [see Eq. (50)] as a corresponding pressure variation. (3) The seismic motion produces a sympathetic vibration in microphone components that results in an anomalous signal. Bedard (1971) discusses the seismic sensitivity for a particular microphone system and concludes that the effect there is negligible. For certain classes of sources, for example earthquakes and surface explosions where the Rayleigh wave amplitude near the microphone is large, care may be needed to differentiate between signals resulting from the direct air transmission and seismic transmission. The high value of trace velocity for a surface-wave generated signal will help to discriminate it from a direct acoustic signal.

\section{SIGNAL DISTORTION}

It is important to ask if the signal produced by a microphone is an accurate representation of the original signal; that is, is $P$ - $p$ exactly proportional to $P_{e}$ ? Considering an original signal decomposed into its Fourier components, we see that each component has its amplitude multiplied by the response, $\mathfrak{R}$, and has a phase lag, $\phi$, applied by the action of the microphone. The modified amplitude components then are recombined to form the resultant output signal. If the response is flat over the domain of the Fourier components and the phase lag is nearly null, there will be little or no distortion. However, in a frequency region of changing response and phase lag, the signal will be distorted to some degree. Obviously this will be true if there are Fourier components in the low-pass or high-pass regions. One method to examine the problem of distortion is to use the numerical integration procedure, which predicts the microphone signal, $P-p$, for comparison with the input signal, $P_{e}$.

\section{MICROPHONE SIGNALS AND CALIBRATION}

We now consider the signal produced by the microphone under the influences of the various parameters. The signal is given as an electrical quantity (usually voltage) through the electronic coupling to the mechanical response of the diaphragm to signal $P_{e}$. The signal $S$ (volts) can be written as

$$
S=P_{e}\left(\frac{P-p}{P_{e}}\right)\left(\frac{\Delta V}{P-p}\right)\left(\frac{\Delta z}{\Delta V}\right)\left(\frac{\Delta E}{\Delta z}\right)
$$

Here $\Delta z$ is the linear displacement of the diaphragm perpendicular to its surface (e.g., at its center), corresponding to the volumetric displacement, $\Delta V$, and $\Delta E$ is the voltage change produced by the 
displacement $\Delta z$. The quantities in (43) are taken as rms values. The second factor is identical to the response $\mathfrak{R}$, and the third factor is identical to $b$ from (3). We define

$$
f=\Delta z / \Delta V
$$

and

$$
s=\Delta E / \Delta z,
$$

where $f$ depends upon the mechanical properties of the diaphragm as discussed in a later section, and $s$ is dependent upon the electronic coupling. Note that $\Re$ may contain the effects of electronic filtering in an actual application. Expression (43) can now be rewritten as

$$
S=P_{e} \Re b f s
$$

It is highly desired that the measurement $S$ be linear in the signal pressure, $P_{e}$. This requires that the factors $\Re, b, f$, and $s$ or their combination be constant over the range of pressure differential and frequency to be measured.

The calibration constant, $C$, of a microphone can be given as

$$
C=S_{c} / P_{c}
$$

where $S_{\mathrm{c}}$ is the calibration signal voltage, and $P_{c}$ is the calibration pressure applied to the microphone; these may be rms or peak-to-peak values. Notice that the value of $C$ will be dependent upon the calibration signal frequency. Now using (46) we can rewrite (47) as

$$
C=\mathfrak{R} b f s
$$

If the quantities in the expression were known to the desired accuracy, the calibration could be determined from (48). The functions $b$ and $s$, however, may be difficult to determine experimentally. As a result, $C$ is conventionally determined by a direct measurement using a calibrated differential pressure source and application of (47). In usual practice, secondary calibration standards are used, and these commonly are piston phones or calibration chambers. Piston phones apply a known differential pressure to the entrance port of the microphone. In the use of calibration chambers the entire microphone is placed inside a chamber in which differential pressures are generated. Calibration sources have also been based upon fan or centrifugal generators, as described by Bedard (1977), and upon the concept of the thermophone, as described 
by Wente (1922). Another simple, but rough, method of calibration is through the rapid change in the elevation of a microphone. The change in atmospheric pressure for an increase in elevation $\Delta h$ is given by

$$
\Delta P=-g \rho \Delta h
$$

where $g$ is the acceleration of gravity and $\rho$ the ambient density at the altitude of the calibration. At standard temperature and pressure the expression is approximately

$$
\Delta P(\mu b a r) \approx-1.08 \Delta h(\mathrm{~cm})
$$

Thus a change in elevation of $10 \mathrm{~cm}$ produces a respectable pressure change of about $11 \mu \mathrm{bars}$. Following a rapid (nearly instantaneous) elevation change, the resulting signal decays rapidly to background. For a bare microphone we have $R=0$ and $P=\Delta P=$ constant. Then, using Eq. (8), we derive the following expression for the measured pressure

$$
P-p=\Delta P \exp (-a q t / v)
$$

where $q$ is defined by (A3) in Appendix A. Expression (51) allows us to determine the time, $T_{e}$, for a decay of the signal by $1 / \mathrm{e}$

$$
T_{e}=v / a q
$$

As an example, $T_{e}=0.71 \mathrm{~s}$ for the standard case. This means that the elevation change must be very fast for (51) to be effective.

Piston phones and chamber calibrators both utilize the principle of a reciprocating piston in a cylinder sinusoidally moving a small volume of air in and out of a container having a much larger volume. This motion produces a sine wave differential pressure whose peak-to-peak amplitude is

$$
A=\gamma \pi r^{2} s P_{a} / V
$$

where $s$ is the stroke of the piston with radius $r$, and $V$ is the larger volume. This expression assumes adiabatic conditions in the calibrator. In order to produce calibration pressures in the microbar domain, the ratio of the larger volume to the piston's displacement must be close to one million. An accurate calibration requires that the local ambient pressure, $P_{a}$, be used in (53) to determine the value of the calibration pressure amplitude. On the other hand, once the calibration 
constant, $C$, is determined for a microphone, it is a constant of the microphone and does not change with altitude. Appendix B contains a discussion of the accuracy of a calibrator.

\section{DIAPHRAGM AND LEAK PHYSICS}

The very small motion of the diaphragm suggests that it behaves as a perfect membrane in its expansion. For a circular membrane of radius $R$ and with inherent tension $T$ and applied uniform pressure $P$, the relation governing the displacement, $z$, perpendicular to the membrane at radius $r$ is

$$
\frac{d^{2} z}{d r^{2}}+\frac{1}{r} \frac{d z}{d r}=-\frac{P}{T}
$$

see, for example, Slater and Frank (1947), or Wente (1917).

The solution of (54) for $z$ is

$$
z(r)=\frac{P\left(R^{2}-r^{2}\right)}{4 T}
$$

Expression (55) can be integrated to give the volume, $\Delta V$, of the diaphragm displacement:

$$
\Delta V=\pi P R^{4} / 16 T
$$

Using (56) we can determine the ratio of $z(r=0)$ to the displacement volume, and from (44) this is simply the quantity $f$ :

$$
f=z(0) / \Delta V=4 / \pi R^{2}
$$

The quantity $f$ is independent of $T$ and thus of the mechanical properties of the diaphragm. Now, using the definition (3) for the diaphragm constant $b$ and (56) we can write

$$
b=\pi R^{4} / 16 T
$$

which provides a definition for $b$ in terms of the diaphragm's physical parameters.

Since the diaphragm has the properties of an acoustic drumhead, it is useful to examine its fundamental frequencies; naturally we would expect such vibrations to be far from the infrasound region. For a circular membrane we can write for the natural frequencies 


$$
\omega=k \beta / 2 R,
$$

where

$$
k=\sqrt{T / \sigma} .
$$

The value $\sigma$ is the areal density of the diaphragm and $\beta$ is a parameter based upon the desired vibrational mode ( $\beta=0.766$ for the fundamental). For $b=10^{-4} \mathrm{cgs}$ and assuming $\sigma=0.003 \mathrm{~g}$ $\mathrm{cm}^{-2}$, the fundamental is about $2500 \mathrm{rad} \mathrm{s}^{-1}$. Higher modes will have higher frequencies. These frequencies are not of consequence for detection of infrasound; however, it is conceivable that induced vibrations of the microphone at these high frequencies could interfere with proper operation.

It is of interest to examine the characteristics of the flow in the leak connecting the fore and backing volumes. The average velocity, $c$, of the flow can be written as

$$
c=\frac{l}{\pi a^{2}} \frac{d V}{d t}
$$

where the quantities have their previous meanings. Using (1) and (2) in (61) gives

$$
c=(P-p) a^{2} / 8 L \eta
$$

and for the standard case this results in

$$
c\left(\mathrm{~cm} \mathrm{~s}^{-1}\right)=0.022(P-p)
$$

for pressures in $\mu$ bars. Thus for typical differential pressures the velocity will be very small, for example, about $0.2 \mathrm{~cm} \mathrm{~s}^{-1}$ for $10 \mu$ bars. The Reynold's number, $R_{e}$, for cylindrical flow is given by

$$
R_{e}=2 a c \rho / \eta
$$

where $\rho$ is the density of the fluid, and other quantities are as previously defined. For the standard case at a differential pressure of $10 \mu \mathrm{bar}$, we find $R_{e}=0.12$. The requirement for laminar flow is $R_{e}<2100$, which is well satisfied by the leak flow. 


\section{ACKNOWLEDGMENTS}

The author is grateful to Douglas ReVelle, Douglas Christie, and Edwin Bullard for their advice, to Larry Auer for assistance with the calculations, and to Ann Mauzy for editing. The work was supported by the Department of Energy Office of Non-Proliferation and National Security.

\section{REFERENCES}

Bedard, A. J., 1971, Journal of Research of the National Bureau of Standards, 75C, 41.

Bedard, A. J., 1977, Fluidics Quarterly, 9, 26.

Daniels, F. B., 1959, Journal of the Acoustical Society of America , 31, 529.

Haak, H. W., and G. J. de Wilde, 1996, Koninklijk Nederlands Meteorologisch Instituut Report WR 96-06.

Hubbard, E. K., and A. J. Bedard, 1969, ESSA Technical Memorandum, ERLTM-WPL 4, Environmental Science Services Administration, Wave Propagation Laboratory, Boulder, Colorado.

Jones, R. V., and S. T. Forbes, 1962, Joumal of Scientific Instruments, 39, 420.

Pierce, A. D., 1981, Acoustics, McGraw-Hill, Inc., New York.

Slater, J. C., and N. H. Frank, 1947, Mechanics, McGraw-Hill, Inc., New York.

Wente, E. C., 1917, Physical Review, 10, 39.

Wente, E. C., 1922, Physical Review, 19, 333. 


\section{APPENDIX A--DERIVATION OF RESPONSE AND PHASE LAG}

To find a solution for the microphone response, we substitute (10) through (12) in expressions (7) and (8). Upon clearing of $e^{i \omega t}$ we obtain

$$
i \omega m=\frac{a(1-m)}{V R}+\frac{a q(n-m)}{V}
$$

and

$$
i \omega n=\frac{a q(m-n)}{v}
$$

where

$$
q=\frac{l}{r}+i \omega b
$$

and

$$
a=\gamma P_{a} .
$$

(A1) and (A2) can be solved for $m$ and $n$ with the results

$$
m=a / F
$$

and

$$
n=\frac{a^{2} q}{F(i \omega v+a q)}
$$

where

$$
F=i \omega V R+a+a q R-\frac{a^{2} q^{2} R}{i \omega v+a q}
$$

Substituting the results (A5) and (A6) into (14), we have

$$
Q=m-n=\frac{a}{F}\left(1-\frac{a q}{i \omega \nu+a q}\right)
$$

or

$$
Q=\frac{i \omega a v}{F(i \omega v+a q)}
$$

Reintroducing the expressions (A3) and (A7) for $q$ and $F$ into (A9) gives

$$
Q=\frac{i \omega a v}{C+i D}
$$

where

$$
C=\frac{a^{2}}{r}-\omega^{2}[R V v+a b R(V+v)]
$$


and

$$
D=\omega\left[a^{2} b+v a+\frac{a R(V+v)}{r}\right] .
$$

The expression (A10) can be shown to be identical to the result given by Burridge (1971). For ease of comparison, we have used the same notation for microphone parameters as employed by Burridge. We find, however, that the derivation by Burridge appears to have typographical errors that make the sequence of his derivation difficult to follow.

(A10) can be written in standard complex form as

$$
Q=\frac{\omega a v D+i \omega a v C}{C^{2}+D^{2}}
$$

Now, since $Q$ is complex, we find its magnitude, $\Re$, by the standard procedure

$$
\mathfrak{K}=\sqrt{Q Q^{*}}
$$

where $Q *$ is the complex conjugate of $Q$. The result is

$$
\mathfrak{R}=\frac{\omega a v}{\sqrt{C^{2}+D^{2}}}
$$

which provides the response function of the microphone and is given as (15) in the text.

The phase lag, $\phi$, of $P$-p with respect to $P_{e}$ can be found from conventional complex analysis as

$$
\tan \phi=Q_{i} / Q_{r}
$$

where $Q_{i}$ and $Q_{r}$ refer, respectively, to the imaginary and real parts of $Q$. Then, using (A13) we have

$$
\tan \phi=C / D
$$

From (A11) and (A12)

$$
\tan \phi=\frac{a-\omega^{2} r R[b(V+v)+V v / a]}{\omega[r(v+a b)+R(V+v)]}
$$


which is given as (18) in the text.

A useful approximation can be made for (A18) where $V>>v$, which is usually true:

$$
\tan \phi \approx \frac{a / \omega R V-\omega g / a}{(g / R V)+l},
$$

where

$$
g=r(a b+v)
$$

Finally, since in general $g / R V \gg>1$, we can rewrite (A19) as

$$
\tan \phi \approx \frac{a}{\omega g}-\frac{\omega R V}{a}
$$

which is Eq. (19) given in the text.

\section{REFERENCES}

Burridge, R., 1971, Geophysical Journal Of Research of the Royal Astronomical Society, 26, 53. 


\section{APPENDIX B --CALIBRATION ACCURACY}

It is useful to consider the constraints upon the accuracy of microphone calibration. Combining expressions (47) and (53) allows us to write for the calibration constant using a pistontype calibrator with an isothermal approximation,

$$
C=S_{c} V / \gamma \pi r^{2} s P_{a}
$$

If each of the factors in the expression is independent of the others and has an Gaussian error distribution, the variance of $C$ can be expressed by

$$
\sigma_{c}^{2}=\sum_{n=1}^{N}\left(\frac{\partial C}{\partial x_{n}}\right)^{2} \sigma_{n}^{2},
$$

where $x_{n}$ represents each of the factors and the $\sigma_{n}$ the corresponding variances. From (B1) and (B2) we have

$$
\left(\frac{\sigma_{c}}{C}\right)^{2}=\left[\frac{\sigma\left(S_{c}\right)}{S_{c}}\right]^{2}+\left[\frac{\sigma(V)}{V}\right]^{2}+4\left[\frac{\sigma(r)}{r}\right]^{2}+\left[\frac{\sigma(s)}{s}\right]^{2}+\left[\frac{\sigma\left(P_{a}\right)}{P_{a}}\right]^{2}
$$

Equation (B3) can be used to estimate the relative importance of each source of error. We ignore here any errors introduced by the microphone itself. As an example, consider a calibrator with the following uncertainties:

1. Accuracy of the voltage measurement, $\sigma\left(S_{c}\right) / S_{c}$, is $1 \%$;

2. The volume $V=10^{5} \mathrm{~cm}^{3}$ and has a measurement accuracy of $3 \mathrm{~cm}^{3}$; this is typical of a laboratory calibrator;

3. The radius of the piston is $0.5 \mathrm{~cm}$ and has an uncertainty of $0.01 \mathrm{~cm}$;

4. The stroke of the piston is $1 \mathrm{~cm}$ with an uncertainty of $0.01 \mathrm{~cm}$;

5. The ambient pressure is $1000 \mathrm{mbar}$ and is measured with an uncertainty of 5 mbar. Note that, if $P_{a}$ is simply taken as an average value for the altitude of calibration, this error may be about five times larger.

Under these conditions (B3) becomes

$$
\left(\frac{\sigma_{c}}{C}\right)^{2}=10^{-4}+9 \times 10^{-30}+16 \times 10^{-4}+10^{-4}+0.25 \times 10^{-4}
$$

or

$$
\sigma_{c} / C=0.04
$$


Thus, for this example a calibrator accuracy of about $4 \%$ can be expected. The dominant source of error, the third term in (B4), is from the uncertainty in the piston radius; therefore considerable care in the measurement of the radius is warranted. An increase in piston radius decreases the calibration uncertainty but correspondingly increases the pressure amplitude unless $V$ also is increased. Another significant source of error may be the leakage of air between the piston and the cylinder. From the previous discussion of leak rates, it can be seen that this may be a problem at very low calibration frequencies where the leak time constant is comparable to the cyclical period.

A different source of error in the calibration process may be from thermally driven motion of the air in the volume $V$. This motion can produce random pressure variation near or above the level of the calibrator amplitude and thus makes the measurement of $S_{c}$ very inaccurate. Albert $J$. Bedard (National Oceanographic and Atmospheric Administration) and others have demonstrated a reduction of this effect by the introduction of steel wool or vermiculite in the reference volume to promote temperature uniformity. This procedure has also been used in the backing volume of some microphones.

An obvious alternative to the use of equation (B1) for calibration is to determine the pressure amplitude of the calibrator by referencing it to a primary differential pressure standard. This eliminates the need to measure the various parameters except for $P_{a}$, and we have

$$
C=S_{c} P_{a} / A P_{c}
$$

where $P_{c}$ is the ambient pressure during calibration and $A$ is the calibrator pressure amplitude at $P_{a}$. This procedure can reduce the error of calibration and perhaps eliminate biases. Unfortunately, there are few readily available sources of primary standards for the microbar region at present. 